\title{
Uterine Corpus Low Grade Endometrial Stromal Sarcoma
}

National Cancer Institute

\section{Source}

National Cancer Institute. Uterine Corpus Low Grade Endometrial Stromal Sarcoma. NCI

Thesaurus. Code C40223.

A rare, indolent, invasive mesenchymal tumor that arises from the endometrial stroma. It

is characterized by the presence of a plexiform vasculature, infrequent mitoses, and insignificant cytologic atypia. Late recurrences may occur. 